\title{
Surface Resonant-States-Enhanced Acoustic Wave Tunneling in Two-Dimensional Phononic Crystals
}

\author{
Manzhu Ke, Zhaojian He, Shasha Peng, Zhengyou Liu, ${ }^{*}$ and Jing Shi \\ Key Lab of Acoustic and Photonic Materials and Devices of Ministry of Education and Department of Physics, \\ Wuhan University, Wuhan 430072, China \\ Weijia Wen and Ping Sheng \\ Department of Physics, The Hong Kong University of Science and Technology, Clear Water Bay, Hong Kong \\ (Received 20 November 2006; published 26 July 2007)
}

\begin{abstract}
We show that by placing a metal plate next to a two-dimensional phononic crystal, acoustic waves can tunnel through the combined structure at a specific frequency that lies inside the band gap of the phononic crystal. The enhanced transmission is attributed to the coupling of the input waves with the acoustically resonant states created between the metal plate and the phononic crystal. Experiments are in excellent agreement with the theoretical predictions.
\end{abstract}

DOI: 10.1103/PhysRevLett.99.044301

PACS numbers: 43.35.+d, 42.79.Dj, 63.20.-e, 73.20.Mf

There is a growing interest in acoustic wave propagation in periodic composites known as phononic crystals (PCs) [1-10]. Great progress has already been made in understanding the nature of wave propagation in PCs, such as band gap formation [1-5], negative refraction [6-9], directional radiation [10], and so on. Extensive PC applications have also been anticipated, for example, in sound insulations and acoustic filters, wave guides, etc. Surface modes and their relevant phenomena have always been an important issue in the study of PCs. On the one hand, band gaps for surface waves can be achieved by creating periodic patterns on the surface of solid materials [11-13]; on the other hand, acoustic wave transmission can be enhanced by surface modes in the band gap of a layered structure, composed of two components with very small impedance mismatch, interfaced to a medium of high impedance. The surface modes in the latter case are not true surface waves, but resonant surface states (RSSs) created at the interface [14]. In this Letter, we report a novel observation of enhanced acoustic wave tunneling in a structure consisting of a two-dimensional (2D) PC accompanied by one or two metal plate(s), placed in water. The PC is a periodic array of steel cylinders. The calculated transmission spectra based on the layered multiple scattering theory (LMST) [15-19] show excellent agreement with the experiment. It is further shown that the enhanced tunneling is due to the coupling of the input acoustic waves with the RSSs localized at the interface(s) of the PC and the metal plate(s). This is a first experimental demonstration of acoustic wave tunneling through $\mathrm{PC}$ in the band-gap regime, through coupling with resonant surface states.

The experimental setup is based on the well-known ultrasonic transmission technique [8,20]. Figure 1 gives a schematic picture showing the position of the sample with respect to the generating transducer and the receiving transducer. Both the generating and receiving transducers have a diameter of $25 \mathrm{~mm}$ and a central frequency of $0.5 \mathrm{MHz}$. The entire assembly is immersed in a water tank. A pulser or receiver generator (Panametrics model 5900PR) produces a short-duration pulse with a width of $200 \mu \mathrm{s}$. The generating transducer is placed far away from the sample to yield an input pulse approximating a plane wave. The input pulse is determined by measuring its amplitude and phase in water without the sample in place. By Fourier-transforming the transmitted and input signals, the amplitude $A(f)$ and the phase $\phi(f)$ ( $f$ denotes frequency) for the transmitted and input waves are obtained. The transmission coefficient is defined by $|T(f)|=$ $A_{\text {tr }}(f) / A_{\text {in }}(f)$, where $A_{\text {tr }}(f)$ and $A_{\text {in }}(f)$ are the amplitudes for the transmitted and the input waves, respectively, at frequency $f$. The phase information can be used to calculate the band structure, the phase and/or group velocities, and the directional density of states.

Theoretical calculations for transmission and field distribution are based on the LMST approach [18,19]. Within

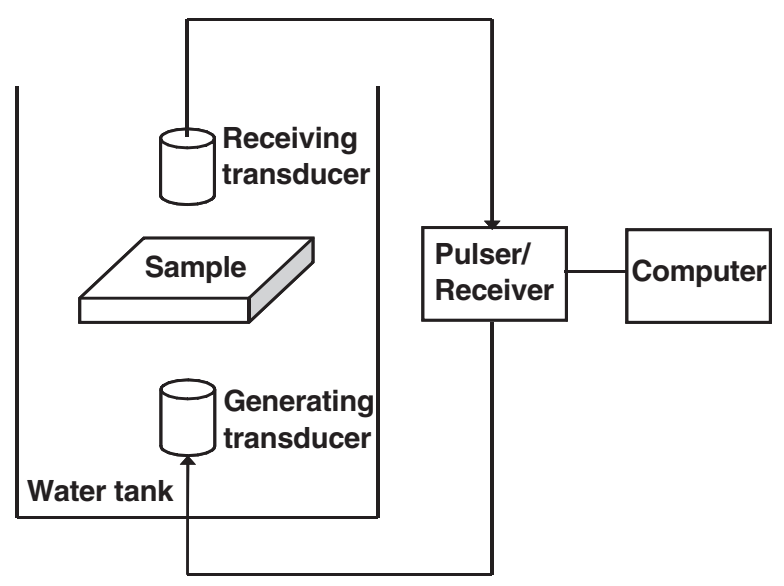

FIG. 1. Schematic representation of the experimental setup. 
the framework of LMST, a PC is regarded as a stacking of layers, periodically arranged along the surface normal. Within each layer the scatters are arranged periodically in accordance with the crystal structure. Since the spherical or cylindrical wave expansions for the field are used in LMST, LMST is ideally suited to calculating the transmission, reflection, and the field distribution for a PC slab consisting of spherical or cylindrical scatterers. In particular, LMST can also easily handle a structure composed of a PC slab accompanied by plates $[15,17]$ placed parallel to the layers.

The 2D PC we used in the experiment consists of an array of solid steel cylinders in a square lattice with a lattice constant $a=1.5 \mathrm{~mm}$. The steel cylinders have a diameter of $1.0 \mathrm{~mm}$. To reveal the effect of the resonant interfacial mode introduced by placing a steel plate on the PC surface, measurements of the acoustic wave transmission were performed for four samples: (a) a steel plate alone, (b) a PC slab alone, (c) a PC slab accompanied by a steel plate on the bottom surface, and (d) a PC slab sandwiched by two steel plates. For all the samples, the PC slab has a thickness of four layers and the steel plate has a thickness of $0.5 \mathrm{~mm}$. The measured results are presented in Figs. 2(a)-2(d), with the corresponding samples schematically shown in the insets. For sample (a), a steel plate alone, the transmission spectrum is typical for acoustic wave transmission through a thin plate. Since the wavelength is much larger than the plate thickness, there is no Fabry-Perot resonance in the frequency region of interest. For sample (b), a PC slab alone, a frequency gap extending from 0.323 to $0.654 \mathrm{MHz}$ can be clearly observed in the transmission spectrum. Theory prediction by the LMST approach shows excellent agreement with the experiment. For sample (c), a PC slab accompanied by a steel plate on the bottom surface, an enhanced tunneling transmission peak, with amplitude about 0.65 , appears at $0.46 \mathrm{MHz}$ that is in the frequency gap of the PC. This feature was also predicted by theory when the steel plate is taken into account. By comparing the transmission spectra of a steel plate alone and a PC slab alone, it is straightforward to deduce that the peak arises from the interaction between the steel plate and the PC slab. To know more about the nature of the peak, one more steel plate was introduced on the top surface of the PC slab [sample (d)]. A splitting of the transmission peak is observed. Calculation based on the LMST approach once again confirms the experimental observation. The splitting of the peak reminds us of the coupling between the two resonant states. We conclude that there exist RSSs at the interface of the PC slab and the steel plate. For a single interface, the RSSs at the single interface, by coupling with the incident waves, give rise to the single (resonant) tunneling peak in the gap. For double interfaces, the coupling of the RSSs at two individual interfaces results in the splitting of the peak.

To have further insight into the tunneling peak arising from the RSSs, the dependencies of the tunneling peak on the separation between the steel plate and the PC slab, on the thickness of the steel plate, and on the thickness of the PC slab are investigated. Figures 3(a1), 3(a2), and 3(a3) show the transmission spectra for a PC slab accompanied by a steel plate on the bottom [see the inset in Fig. 2(c)] with separation between the steel plate and the PC slab being $0.1,0.3$, and $0.6 \mathrm{~mm}$, respectively. It can be seen that the tunneling peak moves to lower frequencies with increasing separation. This feature can be easily understood because the RSSs are closely related to the interface structure. With a further increase in separation, the peak finally moves out of the band gap, indicating the disappearance of

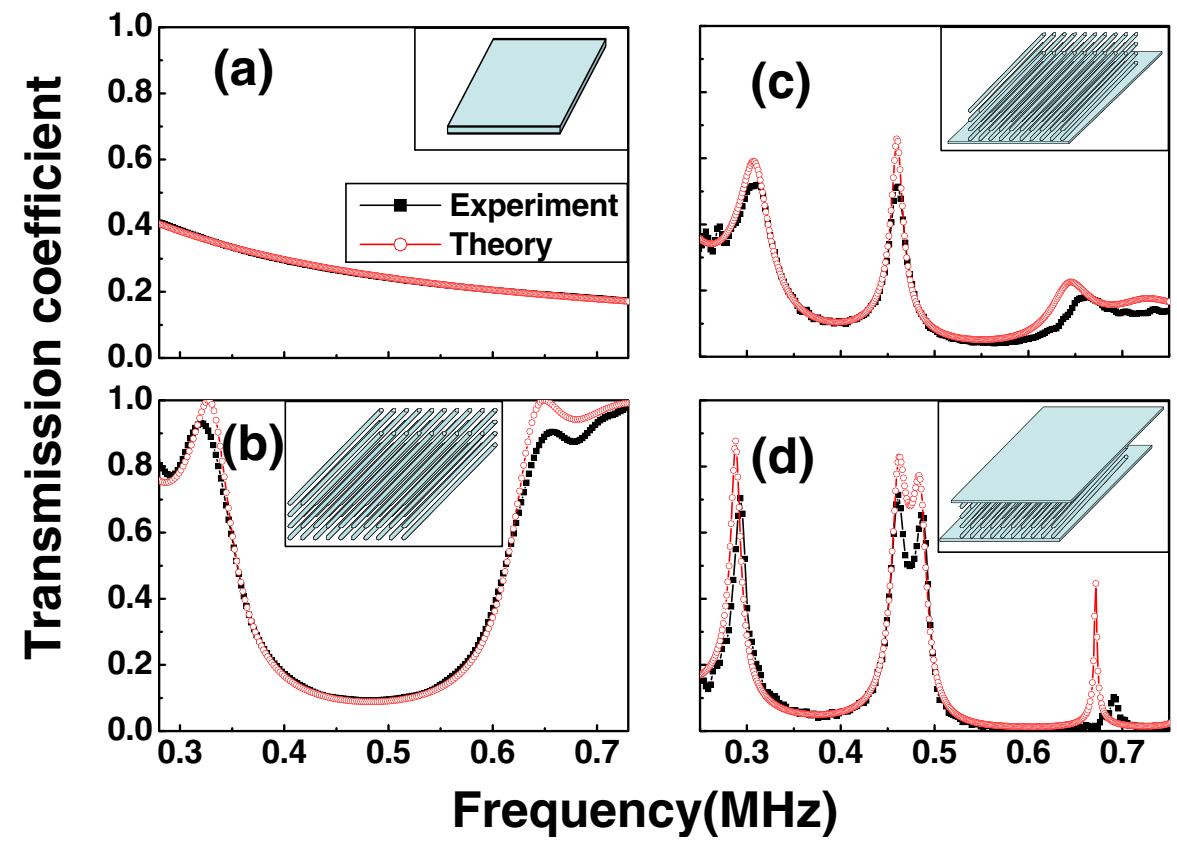

FIG. 2 (color online). Transmission spectra of acoustic waves through (a) a steel plate alone, (b) a PC slab alone, (c) a PC slab accompanied by a steel plate on the bottom, and (d) a PC slab sandwiched by two steel plates. Solid squares denote experimental data; open circles denote theory predictions. The insets give schematic representations of corresponding sample configurations. 

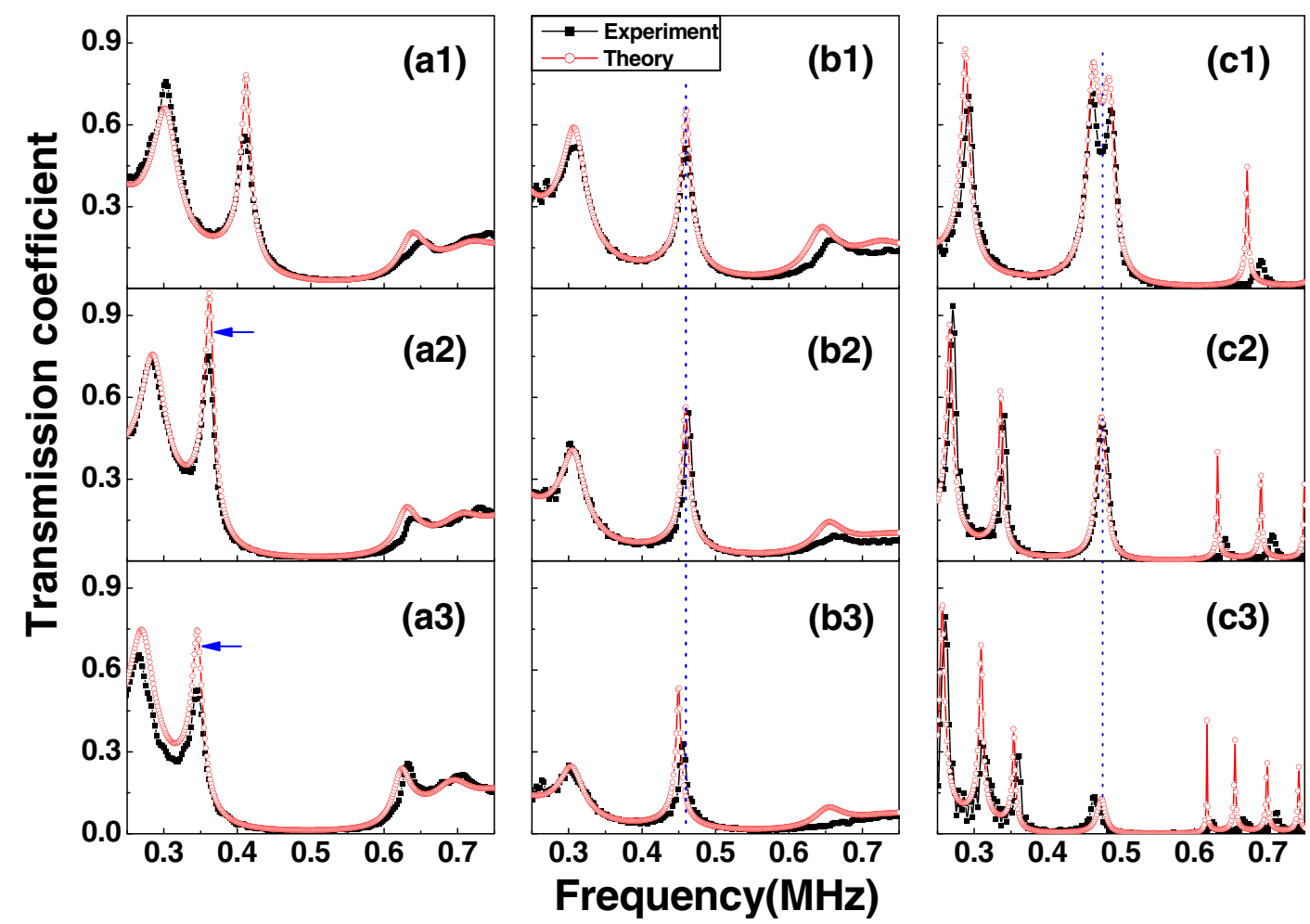

FIG. 3 (color online). Transmission spectra of (a) a PC slab accompanied by a steel plate on the bottom but with different slab-plate separations: (a1) $0.1 \mathrm{~mm}$, (a2) $0.3 \mathrm{~mm}$, (a3) $0.6 \mathrm{~mm}$; (b) a PC slab accompanied by a steel plate on bottom, but with different plate thickness: (b1) $0.5 \mathrm{~mm}$, (b2) $0.8 \mathrm{~mm}$, (b3) $1.5 \mathrm{~mm}$; (c) a PC slab sandwiched by two steel plates on top and on bottom, but with different PC slab thickness: (c1) 4 layers, (c2) 6 layers, (c3) 8 layers. Again, solid squares denote experimental data while open circles denote theory.

the RSSs. Figures 3(b1), 3(b2), and 3(b3) give the transmission spectra for a similar structure as above, but with varying steel plate thicknesses of $0.5,0.8$, and $1.5 \mathrm{~mm}$. It shows the tunneling peak to be hardly influenced by the metal plate thickness. This feature can be understood if the RSSs are tightly bound in the water area between the steel plate and the PC slab, hence hardly influenced by the steel plate thickness. Figures 3(c1), 3(c2), and 3(c3) show the transmission spectra for PC slabs sandwiched by two steel plates on top and on bottom [see the inset of Fig. 2(d)] with varying slab thicknesses: 4,6 , and 8 layers. We see that with increasing PC thickness the two split tunneling peaks merged into one peak, indicating the disappearance of coupling between the RSSs at the two individual interfaces.

To demonstrate the existence of the RSSs, we have calculated the directional density of states (DDOS) along the surface normal of the sample shown in the inset of Fig. 2(c). The DDOS is proportional to $d k / d \omega$, where $\omega$ is the angular frequency and $k$ the wave number. As the phase shift $\phi$ of a wave through a sample is proportional to the wave number in the sample, the DDOS is thus proportional to $d \phi / d \omega$. With a plane wave incident onto the sample, the transmitted waves, with the amplitude and the phase shift $\phi$, can be calculated with the LMST method. The DDOS thus can be obtained by calculating $d \phi / d \omega$. Figure 4 shows the DDOS obtained by this method. We observe a very high DDOS at $0.46 \mathrm{MHz}$, which is a direct evidence of the RSSs with a high density of states. To have a more direct demonstration of the RSSs, we also calculate the field distribution throughout the sample subject to an incident plane wave at frequency $0.46 \mathrm{MHz}$, by using the

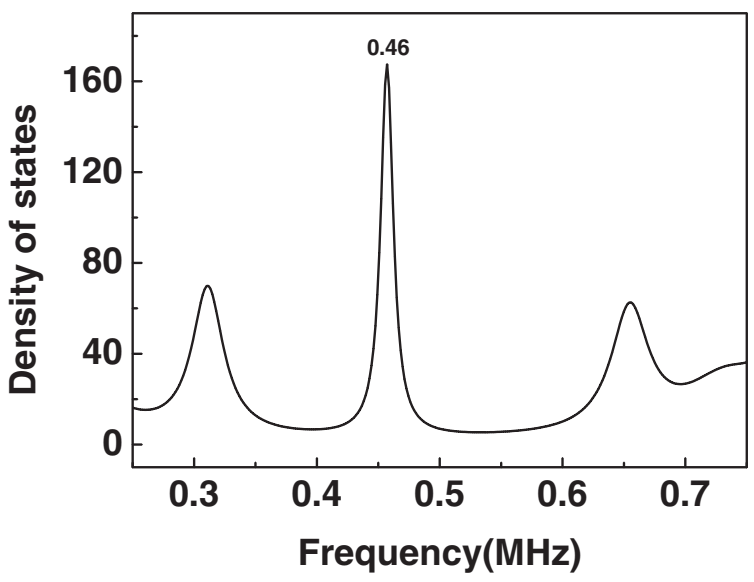

FIG. 4. Calculated DDOS (in arbitrary unit) along the surface normal direction of the sample shown in the inset of Fig. 2(c). 

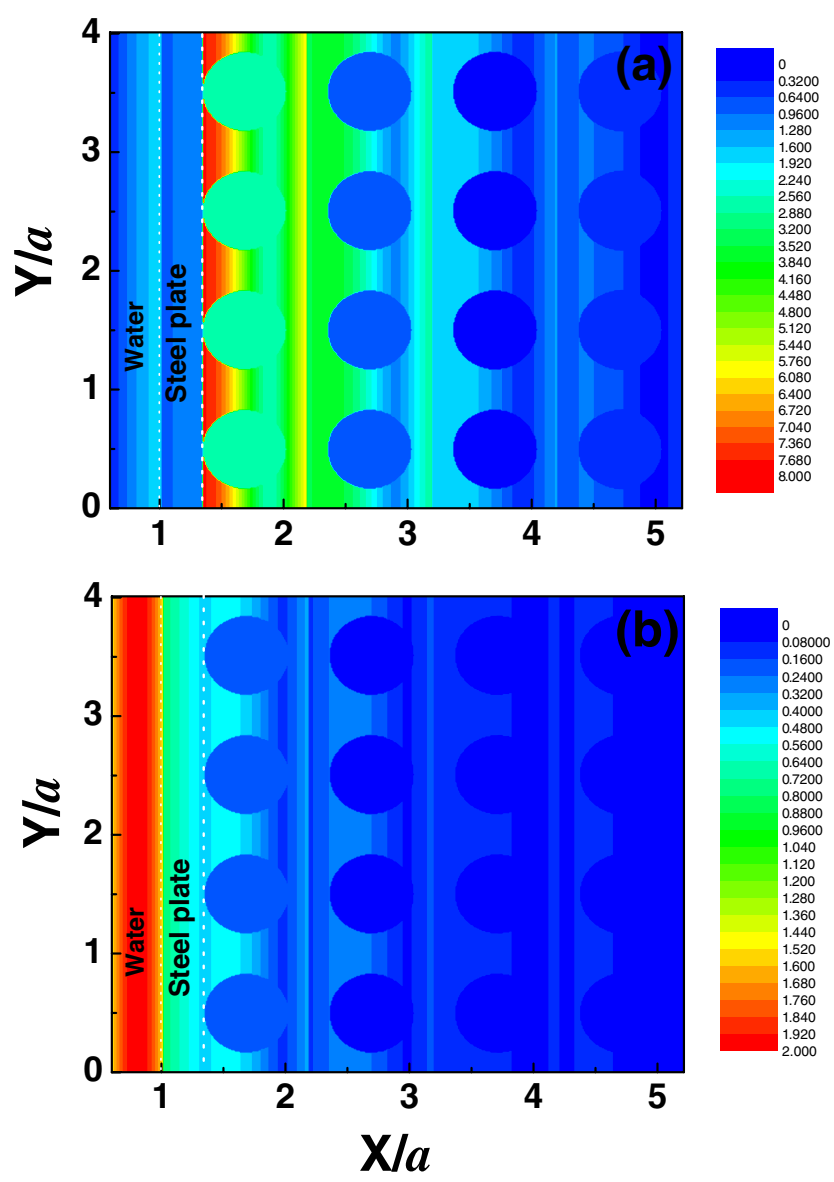

FIG. 5 (color online). Calculated displacement field distribution throughout the sample shown in the inset of Fig. 2(c), subject to an incident plane wave of frequency (a) $0.46 \mathrm{MHz}$, the frequency of the resonant surface states, (b) $0.55 \mathrm{MHz}$, out of the frequency of the RSSs. From left to right, are shown water, steel plate, and PC slab of four layers (the steel cylinders in the PC have clear boundaries), as marked by the vertical dashed lines. The axes are in units of $a$, the lattice constant of the PC.

LMST method. As shown in Fig. 5(a), there is a very strong field distribution at the interface between the steel plate and the PC slab, which is direct evidence for the RSSs. In contrast, in Fig. 5(b) we show the field distribution throughout the sample subject to an incident wave with frequency $0.55 \mathrm{MHz}$, out of the frequency of the RSSs. It can be seen that the field distribution at the interface at this frequency is much weaker, indicating the absence of RSSs.

In conclusion, acoustic wave resonant tunneling through a PC slab accompanied by metal plates within the frequency gap of the PC is demonstrated both experimentally and theoretically. It is found that resonant tunneling can be attributed to the coupling of the incident waves with the resonant surface states at the interface of the PC slab and the steel plate. From all the evidence, a very simple physical picture emerges for the RSS - it is due to the total reflection of the PC (in the gap frequency regime) on the one side, and the near-total reflection of the metal plate on the other. When the metal plate surface coincides with the nodal plane of the standing wave that results from the incident and the totally reflected wave from the PC, a resonant surface state emerges. It follows from this physical interpretation that surface guided modes are potentially possible. Studies in pursuit of these interesting modes are currently in progress.

This work is supported by the National Natural Science Foundation of China (Grants No. 50425206 and No. 10418014).

\footnotetext{
*zyliu@whu.edu.cn
}

[1] M. S. Kushwaha, P. Halevi, L. Dobrzynski, and B. DjafariRouhani, Phys. Rev. Lett. 71, 2022 (1993).

[2] J. O. Vasseur, P. A. Deymier, B. Chenni, B. DjafariRouhani, L. Dobrzynski, and D. Prevost, Phys. Rev. Lett. 86, 3012 (2001).

[3] M. Kafesaki, M. M. Sigalas, and N. Garcia, Phys. Rev. Lett. 85, 4044 (2000)

[4] M. Torres and F. R. Montero, Ultrasonics 42, 787 (2004).

[5] D. Garcia-Pablos, M. Sigalas, F. R. Montero de Espinosa, M. Torres, M. Kafesaki, and N. Garcia, Phys. Rev. Lett. 84, 4349 (2000).

[6] X.D. Zhang and Z. Y. Liu, Appl. Phys. Lett. 85, 341 (2004).

[7] M.Z. Ke, Z. Y. Liu, C. Y. Qiu, W. G. Wang, J. Shi, W. J. Wen, and P. Sheng, Phys. Rev. B 72, 064306 (2005).

[8] S. X. Yang, J. H. Page, Z. Y. Liu, M. L. Cowan, C. T. Chan, and P. Sheng, Phys. Rev. Lett. 93, 024301 (2004).

[9] L. Feng, X. P. Liu, M. H. Lu, Y. B. Chen, Y. F. Chen, Y. W. Mao, J. Zi, Y. Y. Zhu, S. N. Zhu, and N.-B. Ming, Phys. Rev. Lett. 96, 014301 (2006).

[10] M.Z. Ke, Z. Y. Liu, P. Pang, W. G. Wang, Z. G. Cheng, J. Shi, X.Z. Zhao, and W. J. Wen, Appl. Phys. Lett. 88, 263505 (2006).

[11] Y. Tanaka and S. Tamura, Phys. Rev. B 58, 7958 (1998); 60, 13294 (1999).

[12] T. T. Wu, Z. G. Huang, and S. Lin, Phys. Rev. B 69, 094301 (2004).

[13] X. Y. Zhang, T. Jackson, E. Lafond, P. Deymier, and J. Vasseur, Appl. Phys. Lett. 88, 041911 (2006).

[14] H. Kato, Phys. Rev. B 59, 11136 (1999).

[15] Z. Liu, C. T. Chan, Ping Sheng, A. L. Goertzen, and J. H. Page, Phys. Rev. B 62, 2446 (2000).

[16] M. Kafesaki and E. N. Economou, Phys. Rev. B 60, 11993 (1999).

[17] I. E. Psarobas, N. Stefanou, and A. Modinos, Phys. Rev. B 62, 278 (2000).

[18] J. Mei, Z. Liu, J. Shi, and D. Tian, Phys. Rev. B 67, 245107 (2003).

[19] C. Y. Qiu, Z. Y. Liu, J. Mei, and M.Z. Ke, Solid State Commun. 134, 765 (2005).

[20] F. R. Montero de Espinosa, E. Jiménez, and M. Torres, Phys. Rev. Lett. 80, 1208 (1998). 